\title{
Evaluation of Various Scoring Systems in Predicting the Outcome in Patients of Perforation Peritonitis
}

\author{
(Drs.) : Keshri Amit*, Umang Singal**, Rajneesh Varshney*** \\ ( *Asst.Prof-Surgery (MS), **SR Surgery (MS ), ***Asst.Prof. - DNB GI-Surgery) \\ Department Of General Surgery, SRMS-IMS, Bhojipura, Bareilly - 243202, UP, India.
}

\begin{abstract}
Aim \& Objectives: Prospective study to evaluate the mortality prediction in patients with perforation peritonitis using APACHE II, SAPS II, MODS, SOFA and MPI scoring systems.

Material and Methods: One hundred patients of hollow viscus perforation peritonitis, from October 2012 June 2014, were evaluated preoperatively using above mentioned scores and outcome noted in terms of mortality; thereafter, accuracy of the five systems in predicting mortality evaluated.

Results: Of the hundred 100 patients, 31 died. Higher age, late presentation, female gender and colonic perforation carried higher mortality; ARDS was the most common complication. It was observed that higher the score, higher was the mortality. More so, APACHE II scores 16-25, SAPS II 40-59, MPI 22-29, MODS 5-8 and SOFA 7-12 correlated significantly $(p<0.5)$ with the predicted outcome.

Conclusion: Among the 5 scoring systems, APACHE II, SAPS II \& MPI showed less observed mortality rate than predicted percentage at lower scores and higher observed mortality rate than predicted percentage at higher scores; whereas MODS and SOFA showed a much higher observed rate as compared to predicted rate, overall. In all the scoring systems there was a linear increase in death rate with increase in scores.
\end{abstract}

Keywords: perforation peritonitis, scoring system, outcome, PDR (predicted death rate).

\section{Introduction}

Peritonitis due to hollow viscus perforation continues to be a common surgical emergency confronting surgeons. Peritonitis secondary to perforation of the gastro--intestinal tract requires emergency surgical intervention and is associated with significant morbidity and mortality. Abdominal sepsis has been a major factor of mortality and since the past few decades, various scoring systems have been developed to prognosticate the outcome in patients of perforation peritonitis. These scoring systems help in stratifying the patients into various risk categories and deciding the treatment plan.

\section{Material \& Methods:}

This study comprised of randomly selected 100 patients of perforation peritonitis admitted at SRMSIMS, Bareilly from October 2012 to June 2014. Ethical clearance was obtained from our institutional Ethical Committee. Written informed consent was obtained from all the patients/their attendants included in the study. Inclusion criteria were patients of perforation peritonitis secondary to hollow viscus perforation, older than 15 years of age, irrespective of sex. Exclusion criteria were patients of perforation peritonitis secondary to abdominal trauma, cases managed conservatively, post-operative peritonitis due to anastomotic leak, peritonitis in pregnant females, and patients of perforated uterus or fallopian tubes. The diagnosis was based on history, clinical examination, pneumo-peritoneum on imaging studies and lastly exploratory laparotomy.

Surgical management of each of these cases was done appropriate to the site of perforation, type and pathology involved. All investigations necessary to obtain the above five scores (APACHE II, SAPS II, MPI, MODS, and SOFA) for each patient was done preoperatively and each score calculated only once preoperatively. Study is irrespective of the ICU stay of the patient.

Post-operatively, complications and mortality were recorded and outcome noted in terms of survivors and non-survivors. The patients were stratified into groups as per criteria of each scoring system. ${ }^{[1][2][3][4][5]}$ Then the observed mortality was compared with the expected morality for each score and p-value calculated for each of the score-groups using MS-Excel.

\section{Results}

Demographic and outcome data are depicted in Table 1. Of the 100 patients, 65 were males and 35 females $(\mathrm{N}=100, \mathrm{~m}=65 \%, \mathrm{f}=35 \%)$. Most of the patients in both groups were below 60 years of age $[\mathrm{m}(<60 \mathrm{y})=57, \mathrm{f}(<60 \mathrm{y})=27]$. None of the 100 patients presented within 24 hrs. of the onset of first symptom; most presented on day 2 (27\%); followed by day $3(24 \%)$. 
Most patients in our study presented with duodenal perforation (including cases of gastric perforation) $[\mathrm{n}=49(49 \%)]$; followed by ileal [24 (24\%)] and thereafter appendicular [(20) 20\%] and others (jejunal and colonic) [(7) 7\%]. Irrespective of the perforation site, males outnumbered females in all groups, except for jejunal perforation. Overall, 31 patients among the 100 died, mostly of elderly age groups and also due to delayed presentation and complications developing in the recovery phase. Survival was more in the younger age groups (100\% in 16-39 years; $57.1 \%$ in 40-59 years). Of the 31 deaths, $16(51.6 \%)$ were females and 15 $(48.4 \%)$ males. Mortality in the colonic perforation group was $100 \%(5 / 5)$; followed by jejunal $50 \%(1 / 2)$. In the group with maximum cases, i.e. duodenal perforation, mortality was 40.8\% (20/49). Of the 20 appendicular perforation cases, all survived (mortality $=0 \%$ ).

Of 69 survivors, 45 patients had an uneventful post-operative period, while all of the non-survivors had one or more complications leading to their deaths (Table 2). Most common complication in survivors was major or minor wound infection or dehiscence (13 patients, $18.8 \% ; 13 \%$ overall). Among non-survivors the major complication was ARDS (27 patients, $87.1 \%$; $27 \%$ overall), followed by septicemia (15\%), leak (12\%) and others.

As depicted in Table 3, based on the predicted death rates, patients by outcome were divided into six APACHE II score groups, three SAPS II groups, five MPI groups, three MODS groups and three SOFA score groups. Finally, predictive death rate accuracy was determined within each sub-group using chi square test and p-values obtained. A linear relationship between mortality rate and scores was observed in all the scoring systems.

In APACHE II scoring system, patients having score between 0 and 10 suffered no mortality while those between 11 and 15 had $33.3 \%$ mortality, and patients with a score of more than 16 had $100 \%$ mortality. There was a significant correlation $(\mathrm{p}<0.05)$ between predicted and observed death rates for scores between 16 and 25.

In SAPS II scoring system, in the first group i.e. patients with scores between 0-39 we observed mortality of 10 patients (13.5\%); in the second group i.e. 40-59 we observed death of 10 patients $(66.6 \%)$; whereas in the last group we had a $100 \%$ mortality rate as against the $76 \%$ PDR. Observed death rates for scores between 40 and 59 were significantly correlating $(\mathrm{p}<0.05)$ with predicted death rate.

Patients having an MPI score of 0-21 suffered no mortality and those with MPI score $=>30$ had a mortality rate of $72.5 \%$. There were 2 mortalities in the score group of 22-29, having 44 patients, which showed a significant correlation $(\mathrm{p}<0.05)$ with the predicted death rate. Patients with MODS Score of $=>5$ suffered a high mortality approaching $100 \%$ which is highly significant (p-value $<0.05$ ). SOFA Score of $<=6$ showed a mortality of $17.9 \%$; whereas score of $=>7$ had a mortality of $100 \%$ which is significant with the expected value $(\mathrm{p}<0.05)$.

\section{Discussion}

Peritonitis remains a hot spot for the surgeons despite advancements in surgical technique and intensive care treatment. Peritonitis, as a disease is a known entity from the days of Hippocrates.

Various factors like age, sex, duration, site of perforation, extent of peritonitis and delay in surgical intervention are associated with morbidity and mortality. A successful outcome depends upon early surgical intervention, source control and exclusive intra-operative peritoneal lavage. Also various methods \& scoring systems are used to identify the risks and morbidity \& mortality in such patients.

$\mathrm{K}$ Bosscha et al did a study on evaluation of the value of various scoring systems (APACHE II, SAPS, Sepsis Severity Score, Multiple Organ Failure, MPI, Ranson and Imrie) in 50 patients. In the univariate analysis, all scoring systems, except Ranson and Imrie, predicted the primary outcome. In the multivariate analysis, only the APACHE II score (hazard ratio 6.7) and the MPI (hazard ratio 9.8) contributed independently to the prediction of outcome. They concluded that combination of APACHE II \& MPI gives the best scores fitting the clinical goals. ${ }^{[1]}$

Borisov et al compared APACHE-II with the Manheim's Peritonitis Index (MPI) and Simplified Acute Physiology Score-II (SAPS-II) scoring system and found it to be most accurate in patients with bacterial peritonitis. ${ }^{[6]}$

Komatsu S et $\mathrm{al}^{[7]}$ in a study of 26 cases between 1996 and 2003, who underwent emergency laparotomy for colorectal perforation, studied the prognostic value of APACHE II, SOFA, MPI, and MOF scores. Concerning the prognostic scoring systems, an APACHE II score of 19, a SOFA score of 8, an MPI score of 30, and an MOF score of 7 or more were significantly related to poor prognosis.

$\mathrm{M}$ Hynninen et $\mathrm{al}^{[8]}$ in a series of 163 consecutive patients with secondary peritonitis, using APACHE II and SOFA scoring system, concluded that the degree of organ dysfunction measured using SOFA score was the best predictor of hospital mortality in patients suffering from secondary peritonitis.

Ochiai $\mathrm{T}$ et $a l^{[9]}$ in a study of 45 patients with colorectal perforation, concluded that patients with a SOFA score $>7$ had a greater risk of hospital death $(\mathrm{p}=0.0085)$. A Billing et $a l^{[10]}$ studied effectiveness of 
MPI score in abdominal sepsis. For patients with a score less than 21, the mean mortality rate was 2.3 (range 011 ) $\%$, for score $21-29,22.5$ (range 10.6-50) $\%$ and for score greater than $29,59.1$ (range $41-87$ ) $\%$.

In our study of 100 patients, the mean age was 39.13 years. There was male preponderance $(65 \%)$ with male to female (35\%) ratio of 1.9:1. The most common etiology of peritonitis was duodenal perforation, seen in $49 \%$ of patients, followed by, ileal (24\%), appendicular (20\%) and others (jejunal and colonic) (7\%). All the patients presented 24 hours after the occurrence of first symptom, generally withpain abdomen. Most of the patients presented on $2^{\text {nd }}$ day $(27 \%)$ followed by day $3(24 \%)$. As the duration of presentation from day of first symptom increased, so did the death rate.

In our study, duodenal perforations included cases with both gastric and duodenal perforations, which was the most common site of perforation (49\%), with a male preponderance and a maximum prevalence in $4^{\text {th }}$ and $5^{\text {th }}$ decades (22\%); ileum was the second most common site of perforation $(24 \%)$, with a male to female incidence ratio of 3:1, most commonly encountered below 60 years of age; the third most common site of perforation is appendicular (20\%), with a male incidence of $70 \%$. It is the most commonly encountered perforation in younger age groups (34.7\%) and was associated with $100 \%$ survival. Other observed perforations were colonic and jejunal with incidence rates of $5 \%$ and $2 \%$ respectively.

In this study of 100 patients, 31 died, with $100 \%$ mortality in patients who were more than 60 years of age. Female sex showed a poorer outcome $(51.6 \%, 16 \%$ overall) as compared to males $(48.4 \%, 15 \%$ overall). Colonic perforation carries a higher risk of mortality (100\%). The most commonly encountered complication is ARDS (32\%, overall) and appears to be one of the most common factors leading to death ( 27 of $32 ; 84.38 \%$ ).

In our study, all the parameters, relevant to all scores, were considered only once at the time of admission and subsequently all the scores were calculated and their values were applied to predict the outcome of the patients before surgery. Also, the duration of ICU stay has not been taken into account to predict the outcome.

In the study, APACHE II Score was calculated at the time of admission, and then on the basis of predicted mortality it was divided into 6 groups. Observed mortality in the group 11-15 was $33.3 \%$ and scores above 16 had a mortality of $100 \%$. All the observed values are significant $(\mathrm{p}<0.005)$. It is in accordance with the previous scores such that as the score increases, mortality also increases.

In the study, SAPS II Score was calculated at the time of admission, and then on the basis of predicted mortality it was divided into 3 groups. In the first group i.e. between $0-39$, mortality of 10 patients $(13.5 \%)$ was observed; in the second group i.e. 40-59, death of 10 patients (66.6\%) was observed; whereas in the last group we had a $100 \%$ mortality rate as against the $76 \%$ PDR. All the observed values are significant $(\mathrm{p}<0.005)$. Results are in accordance with previous studies i.e. with an increase in SAPS II SCORE, the chance of mortality increases.

In the study, MPI Score was calculated at the time of admission, and then on the basis of predicted mortality it was divided into 5 groups. Patients having score between 0-21 suffered no mortality and those with MPI score $=>30$ had a mortality rate of $72.5 \%$. All the observed values are significant $(\mathrm{p}<0.005)$. Results are in accordance with previous studies i.e. with an increase in MPI SCORE, the chance of mortality increases.

In the study, MODS Score was calculated at the time of admission, and then on the basis of predicted mortality it was divided into 7 groups. Patients with MODS Score of $=>5$ suffered a high mortality approaching $100 \%$.

In the study, SOFA Score was calculated at the time of admission, and then on the basis of predicted mortality it was divided into 6 groups. SOFA SCORE of $<=6$ showed a mortality of $17.9 \%$; whereas score of $=>7$ had a mortality of $100 \%$.

Among the five scoring systems, APACHE II, SAPS II \& MPI showed less observed mortality rate than predicted percentage at lower scores and higher observed mortality rate than predicted percentage at higher scores; whereas MODS and SOFA showed a much higher observed rate as compared to predicted rate. In all the scoring systems there was a linear increase in death rate with increase in scores.

The shortcomings in my study were that ICU stay of patients was not taken into account; scores were calculated only once pre-operatively. Also, no randomization was done, as, the first one hundred patients who were operated upon at SRMS-IMS in the given time period were included in the study.

\section{Conclusion}

In all the scoring systems there was a linear increase in death rate with increase in scores. There was a stronger correlation between observed and predicted mortality rates in APACHE II, SAPS II and MPI, thereby indicating their greater usefulness in predicting the outcomes. We observed that all the five scoring systems can be used for assessment of group outcome in patients with peritonitis due to hollow viscus perforation; however it does not provide sufficient confidence for outcome prediction in individual patients. Also further studies need to be undertaken with a higher number of patients to get a better correlation between predicted and observed outcome. 
Author Disclosure Statement: The authors declare that they have no conflict of interest or source of support.

Abbreviations:

APACHE II -Acute Physiology \& Chronic Health Evaluation

ARDS - Acute Respiratory Distress Syndrome

ICU - Intensive Care Unit

MODS - Multiple Organ Dysfunction Score

MPI - Mannheim Peritonitis Index

PDR - Predicted Death Rate

SAPS II - Simplified Acute Physiology Score

SOFA - Sequential Organ Failure Assessment

\section{References}

[1]. K Bosscha, K Reijnders, P F Hulstaert, AAlgra, C Van Der Werken. Prognostic scoring systems to predict outcome in peritonitis and intra-abdominal sepsis. British Journal of Surgery 1997; 84(11):1532-34.

[2]. Knaus WA et al. APACHE II : A severity of disease classification system. Crit Care Med. 1985;13:818-29.

[3]. Vincent JL, de Mendonça A, Cantraine F, et al. Use of the SOFA score to assess the incidence of organ dysfunction/failure in ntensive care units: results of a multicenter, prospective study. Working group on "sepsis-related problems" of the European Society of Intensive Care Medicine. Crit Care Med. 1998;26(11):1793-800. PMID 9824069.

[4]. Marschall JC et al. Multiple Organ Dysfunction Score : a reliable descriptor of a complex clinical outcome. Crit Care med.1995;23:1638-52.

[5]. Le Gall JR et al. A new simplified acute physiology score (SAPS II) based on a European / North American multicenter study.JAMA. $1993 ; 270: 2957-63$

[6]. Borisov D, Kirov M, Kuzkov V, Uvarov D, Nedashkovsky E. A validation of different prognostic scoring systems in the prediction of outcome in peritonitis. Critical Care 2005, 9(Suppl 1):p 224

[7]. Komatsu S, Shimomatsuya T, Nakajima M, Amaya H, Kobuchi T, Shiraishi S, Konishi S, Ono S, Maruhashi K. Prognostic factors and scoring system for survival in colonic perforation.Hepatogastroenterology. 2005 May-Jun;52(63):761-4.

[8]. M. Hynninen, J. Wennervirta, A. Leppäniemi, V. Pettilä. Organ dysfunction and long term outcome in secondary peritonitis.Langenbeck's Archives of Surgery. January 2008;393(1):81-86.

[9]. Ochiai T, Hiranuma S, Takiguchi N, Ito K, Kawaguchi A, Iwai T, Arii S. SOFA score predicts postoperative outcome of patients with colorectal perforation. Hepato-gastroenterology. 2004;51(58):1007-1010.

[10]. Billing, D. Fröhlich. Prediction of outcome using the Mannheim peritonitis index in 2003 patients. BJS. February 1994;81(2):209-213.

Tables:

Table \#1: Demography \& outcome

\begin{tabular}{|c|c|c|c|c|c|c|c|c|c|c|c|c|c|c|c|}
\hline \multirow{3}{*}{ है। } & \multirow{3}{*}{$\frac{\text { Ane Grous }}{\text { (vra) }}$} & \multirow{3}{*}{$\begin{array}{c}\text { Sex } \\
\text { Outeome }\end{array}$} & \multicolumn{10}{|c|}{ Site of Perforation } & \multirow{2}{*}{\multicolumn{2}{|c|}{ (by sex) }} & \multirow{3}{*}{ Total (by Age) } \\
\hline & & & \multicolumn{2}{|c|}{$D$} & \multicolumn{2}{|c|}{ J } & \multicolumn{2}{|c|}{ I } & \multicolumn{2}{|c|}{ A } & \multicolumn{2}{|c|}{ c } & & & \\
\hline & & & $\mathrm{s}$ & ns & $\mathrm{s}$ & NS & $\mathrm{s}$ & NS & $\mathrm{s}$ & NS & 8 & NS & $\mathbf{s}$ & NS & \\
\hline \multirow{2}{*}{ I } & \multirow{2}{*}{16.39} & M & 10 & o & o & $o$ & 14 & o & 12 & o & o & $o$ & 36 & $o$ & \multirow{2}{*}{49} \\
\hline & & $\mathrm{F}$ & 6 & o & o & o & 2 & o & $\mathbf{s}$ & o & o & $o$ & 13 & o & \\
\hline \multirow{2}{*}{2} & \multirow{2}{*}{$40-59$} & $M$ & 10 & 6 & o & $o$ & 2 & $o$ & 2 & $o$ & $\circ$ & I & 14 & 7 & \multirow{2}{*}{35} \\
\hline & & $\mathrm{F}$ & 3 & 3 & I & 1 & 1 & 3 & 1 & o & o & , & 6 & 8 & \\
\hline \multirow{2}{*}{3} & \multirow{2}{*}{60.69} & $M$ & o & o & o & $o$ & 0 & 2 & $o$ & o & o & $o$ & o & 2 & \multirow{2}{*}{5} \\
\hline & & $F$ & $o$ & 3 & o & $o$ & $o$ & $o$ & o & o & o & o & $o$ & 3 & \\
\hline \multirow{2}{*}{4} & \multirow{2}{*}{$70-79$} & M & $o$ & 2 & o & o & o & o & $o$ & o & o & 1 & $o$ & 3 & \multirow{2}{*}{ s } \\
\hline & & $r$ & $o$ & 1 & o & $o$ & $o$ & o & $o$ & o & o & 1 & $o$ & 2 & \\
\hline \multirow{2}{*}{$\mathrm{s}$} & \multirow{2}{*}{$=-80$} & $M$ & o & 2 & o & o & o & o & $o$ & o & o & 1 & o & 3 & \multirow{2}{*}{ o } \\
\hline & & $r$ & o & 3 & o & $o$ & $o$ & $o$ & $o$ & $o$ & o & $o$ & $o$ & 3 & \\
\hline \multicolumn{3}{|c|}{ Totul (by Site/Survivor) } & 29 & 20 & 1 & 1 & 19 & $s$ & 20 & o & o & $s$ & \multirow{2}{*}{69} & & \multirow{2}{*}{100} \\
\hline & & & & \multicolumn{2}{|r|}{2} & \multicolumn{2}{|c|}{24} & \multicolumn{2}{|c|}{20} & \multicolumn{2}{|r|}{$y_{5}$} & & & \\
\hline
\end{tabular}

* Site of Perforation: D-Duodenal (+ Gastric)J-JejunalI-Ileal A-Appendicular C-Colonic * Sex: MMale, F-Female *Outcome: S-Survived, NS-Not Survived

Table \#2: Complications

- Few of the patients had multiple complications 


\begin{tabular}{|c|c|c|c|c|c|c|c|c|c|}
\hline Complications & 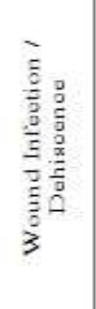 & 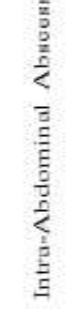 & 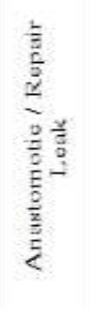 & $\stackrel{\substack{\alpha \\
\theta}}{<}$ & 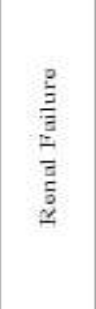 & 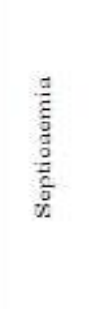 & 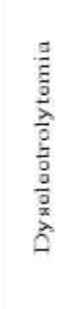 & 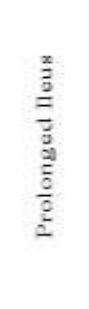 & $\frac{0}{6}$ \\
\hline $\begin{array}{l}\text { Survivors } \\
(\mathbb{1}=69)\end{array}$ & $\begin{array}{c}13 \\
(18.84)\end{array}$ & $\begin{array}{c}2 \\
(2990)\end{array}$ & $\stackrel{2}{2.94}$ & $\begin{array}{c}5 \\
(0.24)\end{array}$ & $0(090)$ & $0(0 \%)$ & $\begin{array}{c}1 \\
(1.5 \%)\end{array}$ & $\begin{array}{c}9 \\
(13.090)\end{array}$ & $\begin{array}{c}45 \\
(65.296)\end{array}$ \\
\hline $\begin{array}{c}\text { Non- } \\
\text { Survivors } \\
(\mathrm{n}=31)\end{array}$ & $\begin{array}{c}10 \\
(32.346)\end{array}$ & $\begin{array}{c}4 \\
(12.9 \%\end{array}$ & $\begin{array}{c}12 \\
(38.796)\end{array}$ & $\begin{array}{c}27 \\
(87.116)\end{array}$ & $\begin{array}{c}11 \\
(35.5 \%)\end{array}$ & $\begin{array}{c}15 \\
(48.486)\end{array}$ & $\begin{array}{c}9 \\
(29.048)\end{array}$ & $\begin{array}{c}4 \\
(12.990)\end{array}$ & $0(098)$ \\
\hline Total & 23 & 6 & 14 & 32 & 11 & 15 & 10 & 13 & 45 \\
\hline
\end{tabular}

Table \#3: Scoring Systems and Observed vs. Predicted Death Rate (PDR)

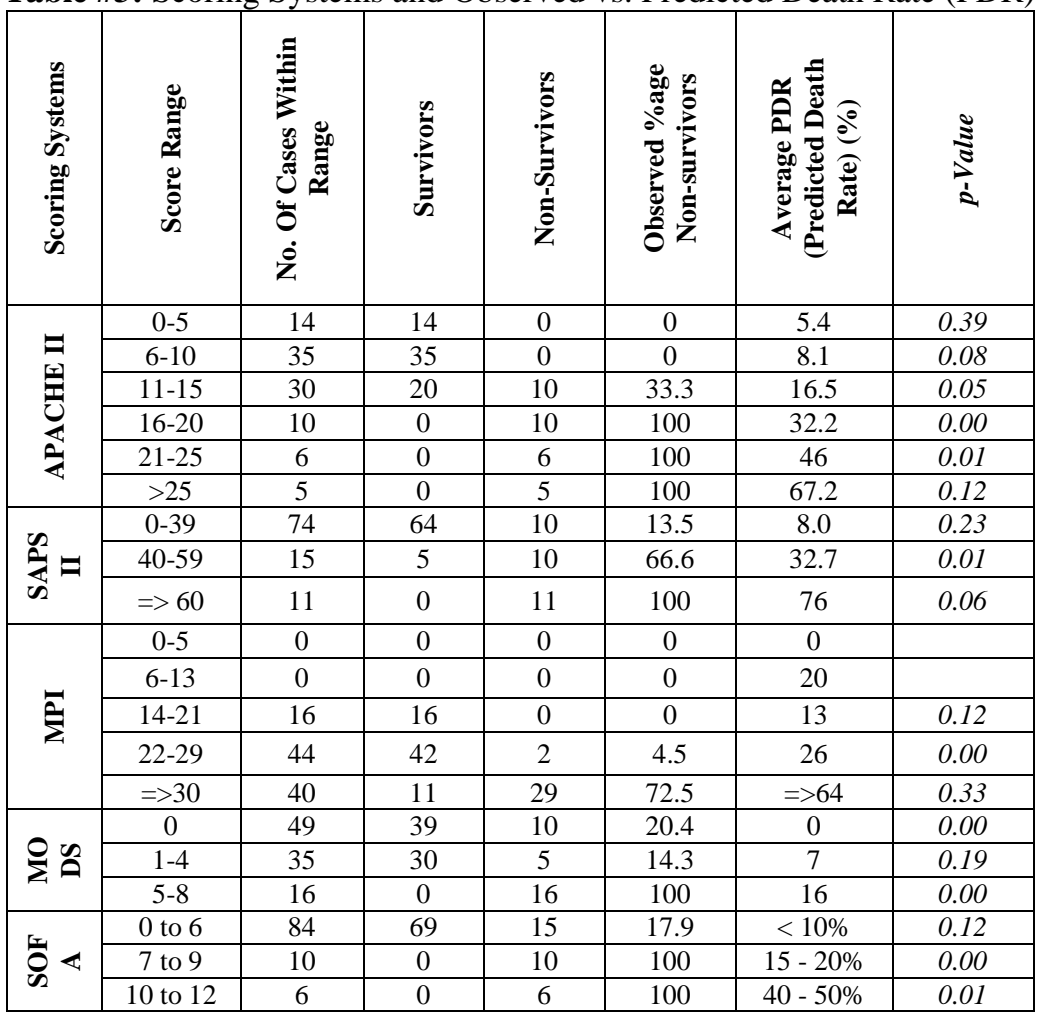

$\mathrm{P}$-value $<0.05$ is taken to be significant

Acknowledgements to Drs. (Dept. Of Surgery): Apurva Gupta, Prof. RK Bajpai, Prof. RK Abbey, Ajay Agarwal, SK Sagar, HarshaVardhan, Firoz Khan, Prateek Shakya.

\&thanks to Faculty, Depts. Of Radiodiagnosis, Clinical Biochemistry, Anesthesiology, Pathology. SRMS-IMS, Bhojipura, Bareilly - 243202, UP, India. 